\title{
Immunohistochemical assessment of mitochondrial superoxide dismutase (MnSOD) in colorectal premalignant and malignant lesions
}

\author{
Adam Piecuch ${ }^{1}$, Marlena Brzozowa-Zasada' ${ }^{1}$ Bartosz Dziewit ${ }^{2}$, Oliwia Segiet ${ }^{1}$, Józef Kurek ${ }^{3}$, \\ Grażyna Kowalczyk-Ziomek ${ }^{1}$, Romuald Wojnicz ${ }^{1}$, Krzysztof Helewski ${ }^{1}$ \\ ${ }^{1}$ Chair and Department of Histology and Embryology, Faculty of Medicine and Dentistry in Zabrze, Medical University of Silesia \\ in Katowice, Poland \\ ${ }^{2}$ Institute of Physics, University of Silesia, Katowice, Poland \\ ${ }^{3}$ Municipal Hospital, Jaworzno, Poland
}

Gastroenterology Rev 2016; 11 (4): 239-246

DOI: 10.5114/pg.2016.57943

Key words: colorectal cancer, colorectal adenoma, manganese superoxide dismutase, immunohistochemistry, oxidative stress.

Address for correspondence: Marlena Brzozowa-Zasada MD, Chair and Department of Histology and Embryology, Faculty of Medicine and Dentistry in Zabrze, Medical University of Silesia, 19 Jordana St, 41-808 Zabrze, Poland, phone: +48 3227228 42, fax: +48 322722867 , e-mail: marlena.brzozowa@op.pl

\begin{abstract}
Introduction: It is generally accepted that mitochondria are a primary source of intracellular reactive oxygen species (ROS). Under physiological circumstances they are permanently formed as by-products of aerobic metabolism in the mitochondria. To counter the harmful effect of ROS, cells possess an antioxidant defence system to detoxify ROS and avert them from accumulation at high concentrations. Mitochondria-located manganese superoxide dismutase (MnSOD, SOD2) successfully converts superoxide to the less reactive hydrogen peroxide $\left(\mathrm{H}_{2} \mathrm{O}_{2}\right)$. To the best of our knowledge, there are no available data regarding immunohistochemical expression of MnSOD in colorectal neoplastic tissues.

Aim: To investigate the immunohistochemical expression status of MnSOD in colorectal premalignant and malignant lesions.

Material and methods: This study was performed on resected specimens obtained from 126 patients who had undergone surgical resection for primary sporadic colorectal cancer, and from 114 patients who had undergone colonoscopy at the Municipal Hospital in Jaworzno (Poland). Paraffin-embedded, 4- $\mu$ m-thick tissue sections were stained for rabbit polyclonal anti SOD2 antibody obtained from GeneTex (clone TF9-10-H10 from America Diagnostica).

Results: Results of our study demonstrated that the development of colorectal cancer is connected with increased expression of MnSOD both in adenoma and adenocarcinoma stages. Samples of adenocarcinoma with G2 and G3 grade showed significantly higher levels of immunohistochemical expression of this antioxidant enzyme. Moreover, patients with the presence of lymphovascular invasion and higher degree of regional lymph node status have been also characterised by higher levels of MnSOD expression. The samples of adenoma have been characterised by higher levels of MnSOD expression in comparison to normal mucosa as well. Interestingly, there was no significant correlation between expression and histological type of adenoma.

Conclusions: Development of colorectal cancer is connected with increased expression of MnSOD both in adenoma and adenocarcinoma stages.
\end{abstract}

\section{Introduction}

It is generally accepted that mitochondria are a primary source of intracellular reactive oxygen species (ROS). Under physiological circumstances they are permanently formed as by-products of aerobic metabolism in the mitochondria [1]. During mitochondrial respiration, the tricarboxylic acid (TCA) cycle generates reducing components acting as a source for electrons.
Electron transfer between mitochondrial electron transport complexes sets up a proton gradient for ATP synthesis [2]. Nevertheless, it has been documented that electrons can flee from the electron transport chain and react with oxygen molecules to form superoxide anions, which is the primary member in a wide array of the ROS family. It is worth mentioning that roughly $1-5 \%$ of the total oxygen consumed during respiration process is 
transformed to superoxide radicals. Superoxide is highly reactive and toxic. It may react with nitric oxide, another free radical generated by mitochondrial nitric oxide synthase, to the highly reactive peroxynitrite. These ROS are major factors responsible for heavy damage to mitochondrial lipids, proteins, and nucleic acids [3-5].

To counter the harmful effect of ROS, cells possess an antioxidant defence system to detoxify ROS and avert them from accumulation at high concentrations [6]. The mitochondria-located manganese superoxide dismutase (MnSOD, SOD2) successfully converts superoxide to the less reactive hydrogen peroxide $\left(\mathrm{H}_{2} \mathrm{O}_{2}\right)$, which may break down further into water and dioxygen by other enzymatic and non-enzymatic antioxidants [7]. Because superoxide primarily emerges from mitochondria, mitochondrial MnSOD is thought to have a fundamental role in ROS detoxification [8].

As revealed by studies, MnSOD plays a very significant role in maintenance of homeostasis in the gastrointestinal tract [9]. To the best of our knowledge, there are no available data regarding immunohistochemical expression of MnSOD in colorectal neoplastic tissues. Colorectal cancer arises from the lesions known as adenomatous polyps, commonly called adenomas. Although every adenoma has the capacity of malignant transformation very few adenomas develop into cancer [10]. A survey comparing adenoma prevalence and carcinoma incidence demonstrated that the percentage of transformation is about $0.25 \%$ per year [11].

\section{Aim}

Therefore, in the present study we investigated the immunohistochemical expression status of MnSOD in colorectal premalignant and malignant lesions. The correlations with significant clinicopathological variables and MnSOD expression in colorectal cancer and adenoma patients were also determined.

\section{Material and methods \\ The group of patients}

This study was performed on resected specimens obtained from 126 patients who had undergone surgical resection for primary sporadic colorectal cancer, and from 114 patients who had undergone colonoscopy at the Municipal Hospital in Jaworzno (Poland). All the specimens were obtained with the consent of the patients. In all cases, a experienced pathologist reviewed the haematoxylin and eosin $(\mathrm{H}+\mathrm{E})$ slides of the adenomas or primary tumours to confirm the pathological features.

The subject population of colorectal cancer patients comprised 83 men and 43 women. The tumours of the patients were classified histopathologically as adeno- carcinoma according to the WHO grading system: grade $1-8$ (6.349\%) patients; grade $2-71$ (56.349\%) patients; grade $3-47$ (37.301\%) patients.

The population of patients with adenoma comprised 53 men and 61 women. The adenomatous polyps were classified as tubular adenomas - 64 (56.140\%) patients; villous adenomas - 28 (24.561\%) patients; and tubulovillous adenomas - 22 (19.298\%) patients.

The design of the study was approved by the ethical committee of the Jerzy Kukuczka Academy of Physical Education in Katowice. The study was supported by grant KNW-1-017/N/3/0 of the Medical University of Silesia.

\section{Immunohistochemistry of MnSOD protein}

Paraffin-embedded, 4- $\mu \mathrm{m}$-thick tissue sections were stained for rabbit polyclonal anti SOD2 antibody obtained from GeneTex (clone TF9-10-H10 from America Diagnostica). Deparaffinisation of all sections was performed through a series of xylene baths, and rehydration was performed through graded alcohols. To retrieve the antigenicity, tissue sections were treated three times with microwaves in a $10 \mathrm{mM}$ citrate buffer ( $\mathrm{pH}$ 6.0) for 5 min each. Subsequently, antigen retrieval sections were incubated with rabbit polyclonal anti-SOD2 antibody (final dilution $1: 400$ ). The En-Vision method (DAKO En-Vision Kit/Alakline Phosphatase detection system) was used according to the manufacturer's instructions. The bound primary antibody was detected using the New Fuchsin Substrate System (DAKO A/S).

\section{Immunohistochemical analysis}

We graded the immunoreactivity by using a semiquantitative approach. Immunohistochemical reaction for MnSOD was classified into four groups according to the intensity of immunohistochemical reaction: 0 , negative; 1 , weak; 2 , moderate; and 3, strong. The intensity of immunohistochemical reaction on the surface epithelial cells has been described as strongly positive. Diffuse staining with the staining intensity weaker than that of surface epithelial cells was characterised as moderately positive. Faint or focal staining was described as weakly positive. Heterogeneity was defined as the proportion of cancer cells showing a positive reaction to the total number of cancer cells and was graded from 0 to 3 by assessment: 0 demonstrated negative staining, 1 represented less than 10\%, 2 represented $10-50 \%$, and 3 represented more than $50 \%$ of cancer cells with positive reaction. The results of intensity of staining and heterogeneity were combined and scored as follows: 0 represented negative, 1 and 2 represented low, 3 and 
4 represented moderate, and 5 and 6 represented high expression.

\section{Statistical analysis}

The relationship between MnSOD expression and clinicopathological variables were examined by the R: A Language and Environment for Statistical Computing (R Core Team., Vienna, Austria) using Pearson's $\chi^{2}$ test. The accepted level of statistical significance was $p<0.05$

\section{Results}

In normal colorectal mucosa MnSOD was predominantly localised in the basal cytoplasm of surface epithelial cells, in which MnSOD immunoexpression was characterised as strong and granular (Figure $1 \mathrm{~A}$ ). In some glandular epithelial cells of the crypts MnSOD expression was also observed. Its intensity was described as weak. Weak expression was also detected in muscularis mucosa and blood vessels in submucosa (Figure $1 \mathrm{~B}$ ). The scattered inflammatory cells localised within lamina propria showed moderate and strong cytoplasmic expression of MnSOD.

In adenoma samples strong granular immunoexpression was detected in neoplastic cells (Figures 1 C, D). In inflammatory cells within lamina propria expression was characterised as moderate or strong. The scattered fibroblast-like cells that were found in close proximity to neoplastic glands were also immunopositive. In those stromal cells, MnSOD immunoexpression was characterised as strong and granular. Adenomas with high grade of dysplasia revealed a significantly higher expression of MnSOD in comparison to those with low grade of dysplasia $\left(\chi^{2}=13.5429\right.$; $\mathrm{d} f=\mathrm{NA}$; $p=0.0004998)$. Moreover, adenomas with high grade of inflammatory infiltration revealed significantly higher expression of this enzyme $\left(\chi^{2}=93.4386\right.$; $d f=7$; $p=2.2 \mathrm{e}-16)$. There was no correlation between intensity of MnSOD immunoreactions and age, gender, localisation, and histological type of adenoma (Table I).

In adenocarcinoma samples, MnSOD expression was found in the cytoplasm of both stromal and cancer cells (Figures 1 E, F). Also, the walls of blood vessels showed positive immunoreactions, but the intensity was weak. The scattered cells with positive reaction were found in submucosa and fatty tissue as well. Immunohistochemical expression of MnSOD in adenocarcinoma samples was significantly correlated with histological grade of tumour $\left(\chi^{2}=19.1451 ; \mathrm{d} f=\mathrm{NA} ; p=0.008496\right)$, lymphovascular invasion $\left(\chi^{2}=16.6624 ; \mathrm{d} f=3 ; p=0.0008292\right)$, and regional lymph node involvement $\left(\chi^{2}=12.9936\right.$; $\mathrm{d} f=\mathrm{NA} ; p=0.04248$ ) (Table II). There was no correlation between MnSOD immunoexpression and age, gender, size of primary tumour, location, and depth of invasion.

\section{Discussion}

Numerous reports have implicated ROS and the activation of redox-sensitive signalling pathways as pivotal players in neoplasm development [12, 13]. Moreover, it has been reported that intrinsic antioxidant enzymes are vital to the regulation of oxidative stress within cells, and alterations of these enzymes are connected with cancer pathogenesis as well [14]. Of these, one of the primary cellular antioxidants SOD catalyses the conversion of superoxide to $\mathrm{H}_{2} \mathrm{O}_{2}$, which can then be removed by catalase, glutathione peroxidases, or peroxiredoxins. In vitro studies have shown that a number of cancer cell lines contain elevated levels of MnSOD and decreased levels of catalase, and that this disruption in steady-state level of $\mathrm{H}_{2} \mathrm{O}_{2}$ showed a correlation with increased metastasis, proliferation, and resistance to apoptosis. MnSOD-dependent production of $\mathrm{H}_{2} \mathrm{O}_{2}$ increased the expression of matrix-degrading metalloproteinases (MMP), which can alter the cancer microenvironment, forming a permissive conditions for metastatic disease $[15,16]$. For example, Hempel et al. demonstrated that acquisition of metastatic phenotype was associated with a significant increase in the expression and activity of MnSOD and decreased activity of catalase. This, in turn, was associated with enhanced steady-state production of $\mathrm{H}_{2} \mathrm{O}_{2}$ in the 253J B-V cells, and with increased activity of MMP-9 and VEGF [17]. Liu et al. determined that MnSOD expression was consistently elevated in patients with tongue cancer. In such patients, expression of MnSOD was significantly higher in cases with lymph node metastases [18]. Several studies demonstrated increased expression of MnSOD in oesophageal [19-24], oral squamous cell carcinoma [25], and pancreatic cancer [26]. In some cases enhanced MnSOD expression was related to poor survival rate or recurrence. Nozoe et al. revealed that the proportion of lymph node metastasis in MnSOD-positive colorectal carcinomas was significantly higher than in MnSOD-negative carcinomas. Moreover, the survival rate in patients with MnSOD-positive carcinomas was worse than that in patients with MnSOD-negative carcinomas [27]. Similar findings have been detected in gastric [19, 28-32] and pancreatic cancer [25], and oral squamous cell carcinoma. The question arises regarding the reasons for enhanced activity of MnSOD in the metastatic stage of the disease. Dhar et al. suggested that reduced Sp1 binding to the MnSOD promoter is responsible for decreased MnSOD expression in the early stages of cancer development, whereas reduced p53 binding activity is responsible for restoration of $\mathrm{MnSOD}$ 

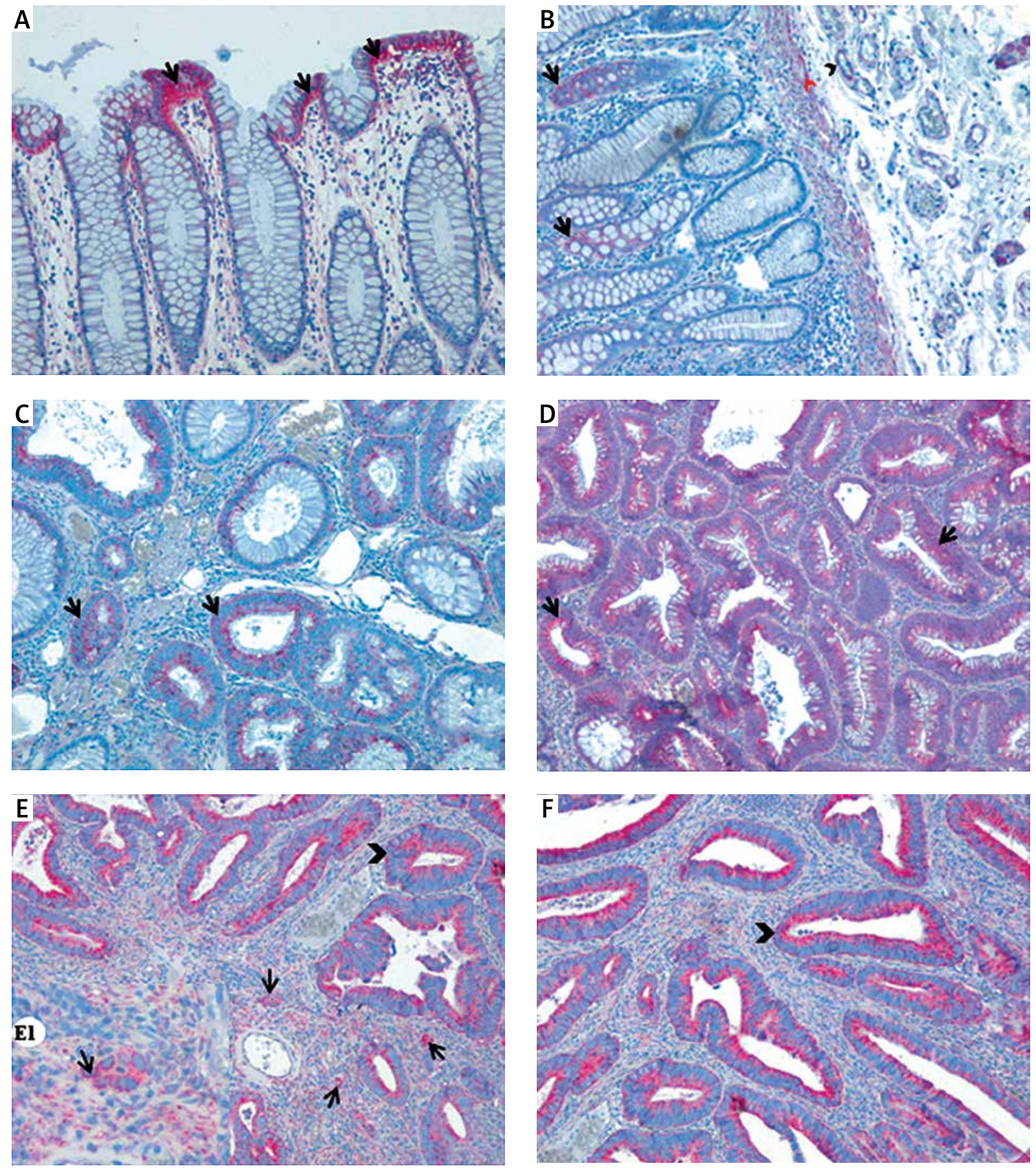

Figure 1. Expression of MnSOD in normal colorectal tissue, colorectal adenomas, and cancer. A - Immunohistochemical reaction in colorectal surface epithelium (arrows). B - Positive immunohistochemical reaction in the colorectal crypts (arrows), muscularis mucosa (arrowhead), and walls of the blood vessels (red arrowhead). C, D - Weak (C) and strong (D) immunoreactions in neoplastic colorectal crypts in adenoma patients. E, F - Positive immunoreaction for MnSOD in colorectal cancer tissues; strong reaction detected in cancerous cells (arrowheads) and stromal cells (E1 - arrows)

at later stages of cancer development. In this case, the balance between Sp1 and p53 plays an important role in regulating the MnSOD level during the phenotyp- ic changes leading to aggressive growth. It has been suggested that increased MnSOD activity may protect cells against mitochondrial injury, thereby conferring 
Table I. Relation between MnSOD expression and clinicopathological variables in colorectal adenoma patients

\begin{tabular}{|c|c|c|c|c|c|c|c|c|c|c|c|}
\hline \multicolumn{2}{|l|}{ Variables } & \multirow{3}{*}{$\begin{array}{c}\text { Total } \\
56\end{array}$} & \multicolumn{8}{|c|}{ Expression status, $n$ (\%) } & \multirow{3}{*}{$\begin{array}{c}\boldsymbol{P} \text {-value } \\
0.1009\end{array}$} \\
\hline & & & \multicolumn{2}{|c|}{ Negative } & \multicolumn{2}{|c|}{ Low } & \multicolumn{2}{|c|}{ Moderate } & \multicolumn{2}{|c|}{ High } & \\
\hline \multirow[t]{2}{*}{ Age } & $<50$ & & 9 & $(16.07)$ & 25 & $(44.64)$ & 16 & $(28.57)$ & 6 & $(10.71)$ & \\
\hline & $\geq 50$ & 58 & 12 & $(20.69)$ & 16 & $(27.59)$ & 27 & $(46.55)$ & 3 & $(5.17)$ & \\
\hline \multirow[t]{2}{*}{ Gender } & Male & 53 & 11 & $(20.75)$ & 16 & (30.19) & 22 & $(41.51)$ & 4 & $(7.55)$ & 0.6647 \\
\hline & Female & 61 & 10 & $(16.39)$ & 25 & $(40.98)$ & 23 & $(37.70)$ & 3 & $(4.92)$ & \\
\hline \multirow[t]{3}{*}{ Location } & Proximal colon & 60 & 10 & $(16.67)$ & 18 & $(30.00)$ & 29 & $(48.33)$ & 3 & $(5.00)$ & 0.07096 \\
\hline & Distal colon & 30 & 5 & $(16.67)$ & 12 & $(40.00)$ & 11 & $(36.67)$ & 2 & $(6.67)$ & \\
\hline & Rectum & 24 & 9 & $(37.50)$ & 11 & $(45.83)$ & 3 & $(12.50)$ & 1 & $(4.17)$ & \\
\hline \multirow{2}{*}{$\begin{array}{l}\text { Inflammatory } \\
\text { infiltrate }\end{array}$} & Weak & 39 & 0 & $(0.00)$ & 8 & $(20.51)$ & 25 & $(64.10)$ & 6 & (15.38) & $<0.001$ \\
\hline & Strong & 75 & 3 & $(4.00)$ & 10 & $(13.33)$ & 21 & $(28.00)$ & 41 & $(54.67)$ & \\
\hline \multirow{2}{*}{$\begin{array}{l}\text { Degree of } \\
\text { dysplasia }\end{array}$} & Low grade & 43 & 1 & $(2.33)$ & 9 & $(20.93)$ & 26 & $(60.47)$ & 7 & $(16.28)$ & 0.0004998 \\
\hline & High grade & 71 & 2 & $(2.82)$ & 17 & $(23.94)$ & 20 & $(28.17)$ & 32 & $(45.07)$ & \\
\hline \multirow{3}{*}{$\begin{array}{l}\text { Histological } \\
\text { type of } \\
\text { adenoma }\end{array}$} & Tubular & 64 & 11 & (17.19) & 19 & $(29.69)$ & 31 & $(48.44)$ & 3 & $(4.69)$ & 0.1719 \\
\hline & Villous & 28 & 5 & $(17.86)$ & 10 & $(35.71)$ & 11 & (39.29) & 2 & (7.14) & \\
\hline & Tubulo-villous & 22 & 7 & (31.82) & 11 & $(50.00)$ & 3 & $(13.64)$ & 1 & $(4.55)$ & \\
\hline
\end{tabular}

a growth advantage to the cancer cells acquiring a more aggressive behaviour [9, 33].

In vitro studies also showed that elevated levels of MnSOD were correlated with acquisition of invasive abilities. It is generally accepted that the major process responsible for cell metastatic behaviour is epithelial mesenchymal transition (EMT) [34]. Interestingly, transcription factors involved in EMT activation seem to be under the influence of MnSOD. Both Snail1 and Snail2 were highly expressed in metastatic UM1 cells in comparison to UM2 cells. Upon MnSOD knockdown in UM1 cells, the intracellular levels of $\mathrm{H}_{2} \mathrm{O}_{2}$ levels were decreased and the expression of Snail factors was inhibited. Moreover, the level of Snail factors was correlated with MMP-1 and ERK1/2. These results may indicate that MnSOD activates Snail signalling, increasing the same metastatic properties of cancer cells [18].

The results of our study remain in agreement with the findings mentioned above. We also revealed that samples of colorectal adenocarcinoma showed significantly higher immunoexpression of MnSOD in comparison to adenomas and adjacent mucosa without any pathological lesions. High intensity of immunoreactions was detected in $\mathrm{G} 3$ tumours, which are known to be highly metastatic. Moreover, expression of MnSOD was correlated with lymphovascular invasion and regional lymph node involvement. Approximately $50 \%$ of patients with lymphovascular invasion showed strong expression of MnSOD, whereas only $15 \%$ from the negative group showed a strong pattern of MnSOD expression. As we discovered, regional lymph node involvement was also correlated with MnSOD positivity. The majority of patients with N2 status showed strong expression, whereas only 1 patient from the NO group showed such strong reaction intensity.

MnSOD expression was also upregulated in the case of colorectal adenomas, which are known as precursor lesions of carcinoma. Adenomas with high grade of dysplasia have significantly higher level of MnSOD immunoexpression. Only 2 patients from the group characterised with a high degree of dysplasia were negative, whereas 45\% demonstrated strong expression. Similar results have been obtained in patients with strong infiltration of immune cells in lamina propria. Half of the cases revealed strong expression, whereas only 3 were described as negative. On the other hand, patients from the group characterised by weak infiltration did not show negative immunoreaction. Most of them showed moderate reaction and only $15 \%$ revealed strong intensity. It is not surprising that inflammatory infiltration is an important factor that can influence MnSOD expression pattern. As revealed by studies, MnSOD is detected not only within cytoplasm of neoplastic cells but also in stromal cells, including macrophages and neutrophils. Accumulating phagocytic leukocytes gener- 
Table II. Relation between MnSOD expression and clinicopathological variables in colorectal cancer patients

\begin{tabular}{|c|c|c|c|c|c|c|c|c|c|c|}
\hline \multirow[t]{2}{*}{ Variables } & \multirow{2}{*}{$\begin{array}{l}\text { Total number } \\
\text { of cases }\end{array}$} & \multicolumn{8}{|c|}{ Expression status, $n$ (\%) } & \multirow[t]{2}{*}{$P$-value } \\
\hline & & \multicolumn{2}{|c|}{ Negative } & \multicolumn{2}{|r|}{ Low } & \multicolumn{2}{|c|}{ Moderate } & \multicolumn{2}{|r|}{ High } & \\
\hline \multicolumn{11}{|l|}{ Age: } \\
\hline$<50$ & 33 & 10 & (30.30) & 4 & $(12.12)$ & 10 & (30.30) & 9 & $(27.27)$ & 0.1654 \\
\hline$\geq 50$ & 93 & 20 & $(21.51)$ & 5 & $(5.38)$ & 23 & $(24.73)$ & 45 & $(48.39)$ & \\
\hline \multicolumn{11}{|l|}{ Gender: } \\
\hline Male & 83 & 17 & $(20.48)$ & 6 & $(7.23)$ & 24 & $(28.92)$ & 36 & $(43.37)$ & 0.2529 \\
\hline Female & 43 & 16 & $(37.21)$ & 3 & (6.98) & 9 & $(20.93)$ & 15 & $(34.88)$ & \\
\hline \multicolumn{11}{|c|}{ Size of primary tumour: } \\
\hline$<5 \mathrm{~cm}$ & 44 & 11 & $(25.00)$ & 0 & $(0.00)$ & 13 & $(29.55)$ & 20 & $(45.45)$ & 0.1844 \\
\hline$\geq 5 \mathrm{~cm}$ & 82 & 22 & $(26.83)$ & 8 & $(9.76)$ & 19 & $(23.17)$ & 33 & $(40.24)$ & \\
\hline \multicolumn{11}{|c|}{ Location of tumour: } \\
\hline Proximal colon & 31 & 3 & $(9.68)$ & 3 & (9.68) & 10 & $(32.26)$ & 15 & $(48.39)$ & 0.2784 \\
\hline Distal colon & 83 & 25 & $(30.12)$ & 7 & $(8.43)$ & 17 & $(20.48)$ & 34 & $(40.96)$ & \\
\hline Rectum & 12 & 3 & $(25.00)$ & 2 & $(16.67)$ & 4 & (33.33) & 3 & $(25.00)$ & \\
\hline \multicolumn{11}{|l|}{ Histological grade: } \\
\hline G3 & 47 & 2 & $(4.26)$ & 3 & (6.38) & 10 & $(21.28)$ & 32 & (68.09) & 0.008496 \\
\hline G2 & 71 & 7 & $(9.86)$ & 4 & $(5.63)$ & 17 & (23.94) & 43 & $(60.56)$ & \\
\hline G1 & 8 & 2 & $(25.00)$ & 3 & (37.50) & 3 & $(37.50)$ & 0 & $(0.00)$ & \\
\hline \multicolumn{11}{|l|}{ Depth of invasion: } \\
\hline $\mathrm{T} 1$ & 6 & 2 & (33.33) & 1 & $(16.67)$ & 2 & (33.33) & 1 & $(16.67)$ & 0.1159 \\
\hline $\mathrm{T} 2$ & 30 & 6 & (20.00) & 7 & (23.33) & 9 & $(30.00)$ & 8 & $(26.67)$ & \\
\hline T3 & 68 & 9 & $(13.24)$ & 15 & (22.06) & 21 & (30.88) & 23 & $(33.82)$ & \\
\hline T4 & 22 & 1 & $(4.55)$ & 9 & $(40.91)$ & 11 & $(50.00)$ & 1 & $(4.55)$ & \\
\hline \multicolumn{11}{|c|}{ Lymphovascular invasion: } \\
\hline Positive & 85 & 10 & $(11.76)$ & 15 & $(17.65)$ & 19 & $(22.35)$ & 41 & $(48.24)$ & 0.0008292 \\
\hline Negative & 41 & 11 & $(26.83)$ & 6 & $(14.63)$ & 18 & $(43.90)$ & 6 & $(14.63)$ & \\
\hline \multicolumn{11}{|c|}{ Regional LN involvement: } \\
\hline N2 & 83 & 21 & $(25.30)$ & 7 & $(8.43)$ & 21 & $(25.30)$ & 34 & $(40.96)$ & 0.04248 \\
\hline N1 & 31 & 1 & (3.23) & 3 & (9.68) & 11 & $(35.48)$ & 16 & $(51.61)$ & \\
\hline NO & 12 & 3 & $(25.00)$ & 2 & $(16.67)$ & 6 & $(50.00)$ & 1 & $(8.33)$ & \\
\hline
\end{tabular}

ates high local concentration of $\mathrm{H}_{2} \mathrm{O}_{2}$ for prolonged time periods [35]. Kruidenier et al. demonstrated that they also contain high MnSOD levels. Interestingly, we did not observe any correlation between MnSOD expression and histological type of adenoma [36]. It is generally accepted that villous polyps in particular are considered high risk. With respect to malignancy, it was found that only $4 \%$ of tubular adenomas but $40 \%$ of villous adenomas developed into carcinoma [37]. It may take about 10 years for an adenomatous polyp to transform into cancer [38].

At this point it is worth noting that increased MnSOD content in aggressive cancer cells may contribute to the repair of mitochondrial function allowing the 
cells to utilise oxidative phosphorylation for maximum energy production to increase cell survival and growth. In the early stages of tumorigenesis, lower MnSOD content may be a threat to mitochondrial function, leading to a shift to glycolysis in cancer cells. Alternatively, it has been proposed that glycolytic metabolism arises as an adaptive response to hypoxic conditions during the early stages of tumour development because it permits ATP generation even in the absence of oxygen. Thus, excess lactate production may further drive the evolvement of glycolytic metabolism for energy production [39]. It has been also proposed that aerobic glycolysis in tumour cells may require rapid influx of substrates through glycolysis, allowing the effective shutting of glucose or fructose as a source of carbon energy production [40-42]. However, because of the consistently increasing demand for energy during the aggressive growth of cancer cells, particularly in the later stages of carcinogenesis, the energy supply from glycolytic processes may not be sufficient. In this situation, it is possible that aggressive cancer cells switch back from glycolysis to oxidative phosphorylation for their increased energy requirements. For this purpose, cancer cells need to increase the efficiency of mitochondrial function [9].

\section{Conclusions}

The results of our study have demonstrated that development of colorectal cancer is connected with increased expression of MnSOD both in adenoma and adenocarcinoma stages. Samples of adenocarcinoma with $G 2$ and $G 3$ grade showed significantly higher levels of immunohistochemical expression of this antioxidant enzyme. Moreover, patients with the presence of lymphovascular invasion and higher degree of regional lymph node status were also characterised by higher level of MnSOD expression. The samples of adenoma were characterised by higher level of MnSOD expression in comparison to normal mucosa as well. Interestingly, there was no significant correlation between expression and histological type of adenoma.

\section{Conflict of interest}

The authors declare no conflict of interest.

\section{References}

1. Kowaltowski AJ, Souza-Pinto NC, Castilho RF, Vercesi AE. Mitochondria and reactive oxygen species. Free Radic Biol Med 2009; 47: 333-43.

2. Dan DJ, Alvarez LA, Zhang X, Soldati T. Reactive oxygen species and mitochondria: a nexus of cellular homeostasis. Redox Biol 2015; 6: 472-85.

3. Keyer K, Gort AS, Imlay JA. Superoxide and the production of oxidative DNA damage. J Bacteriol 1995; 177: 6782-90.
4. Brand MD, Affourtit C, Esteves TC, et al. Mitochondrial superoxide: production, biological effects, and activation of uncoupling proteins. Free Radic Biol Med 2004; 37: 755-67.

5. Pervaiz S, Clement MV. Superoxide anion: oncogenic reactive species? Int J Biochem Cell Biol 2007; 39: 1297-304.

6. Sies H. Strategies of antioxidant defense. Eur J Biochem 1993; 215: 213-9.

7. Zelko IN, Mariani TJ, Folz RJ. Superoxide dismutase multigene family: a comparison of the CUZn-SOD (SOD1), Mn-SOD (SOD2), and EC-SOD (SOD3) gene structures, evolution, and expression. Free Radic Biol Med 2002; 33: 337-49.

8. Imlay JA. Cellular defense against superoxide and hydrogen peroxide. Annu Rev Biochem 2008; 77: 755-76.

9. Dhar SK, Clair DK. Manganese superoxide regulation and cancer. Free Radic Biol Med 2012; 52: 2209-22.

10. Świątkowski M, Medre A, Sobczyński L. Adenomas detected during screening colonoscopies in the years 2000-2009. Prz Gastroenterol 2012; 7: 299-305.

11. Tsao JL, Tavare S, Salovaara R, et al. Colorectal adenoma and cancer divergence. Evidence and multilineage progression. Am J Pathol 1999; 154: 1815-24.

12. Schieber M, Chandel NS. ROS function in redox signaling and oxidative stress. Curr Biol 2014; 24: 453-62.

13. Starkov AA. The role of mitochondria in reactive oxygen species metabolism and signaling. Ann N Y Acad Sci 2008; 1147: 37-52.

14. Ray PD, Huang BW, Tsuji Y. Reactive oxygen species (ROS) homeostasis and redox regulation in cellular signaling. Cell Signal 2012; 24: 981-90.

15. Connor KM, Hempel N, Nelson KK, et al. Manganese superoxide dismutase enhances the invasive and migratory activity of tumor cells. Cancer Res 2007; 67: 10260-7.

16. Kattan Z, Mining V, Leroy P, et al. Role of manganese superoxide dismutase on growth and invasive properties of human estrogen-independent breast cancer cells. Breast Cancer Res Treat 2008; 108: 203-15.

17. Hempel N, Ye H, Abessi B. Altered redox status accompanies progression to metastatic human bladder cancer. Free Radic Biol Med 2009; 46: 42-50.

18. Liu Z, Li S, Cai Y, et al. Manganese superoxide dismutase induces migration and invasion of tongue squamous cell carcinoma via H202-dependent Snail signaling. Free Radic Biol Med 2012; 53: 44-50.

19. Izutani R, Asanom S, Imano M, et al. Expression of superoxide dismutase in esophageal and gastric cancers. J Gastroenterol 1998; 33: 816-22.

20. Hermann B, Li Y, Ray MB, et al. Association of manganese superoxide dismutase expression wit progression of carcinogenesis in Barret esophagus. Arch Surg Oncol 2007; 14: 2045-55.

21. Li Y, Wo JM, Su RR, et al. Alterations in manganese superoxide dismutase expression in the progression from reflux esophagitis to esophageal adenocarcinoma. Ann Surg Oncol 2007; 14: 2045-55.

22. Izutani R, Kato M, Assano S, et al. Expression of manganese superoxide dismutase influences chemosensitivity in esophageal and gastric cancer. Cancer Detect Prev 2002; 26: 213-21. 
23. Sun GG, Wang YD, Lu YF, Hu WN. Different association of manganese superoxide dismutase gene polymorphism with risk of prostate, esophageal, and lung cancers: evidence from meta-analysis of 20,025 subjects. Asian Pacific J Cancer Prev 2013; 14: 1937-43.

24. Janssen AM, Bosman CB, van Duijn W, et al. Superoxide dismutase in gastric and esophageal cancer and the prognostic impact in gastric cancer. Clin Cancer Res 2000; 6: 3183-92.

25. Ye $\mathrm{H}$, Wang A, Lee BS, et al. Proteomic based identification of manganese superoxide dismutase 2 (SOD2) as a metastasis marker for oral squamous cell carcinoma. Cancer Gen Prot 2008; 5: 85-93.

26. Lewis A, Du J, Liu J, et al. Metastatic progression of pancreatic cancer: changes in antioxidant enzymes and cell growth. Clin Exp Metastasis 2005; 22: 523-32.

27. Nozoe T, Honda M, Inatsuka S, et al. Significance of immunohistochemical expression of manganese superoxide dismutase as a marker of malignant potential in colorectal carcinoma. Oncol Rep 2003; 10: 39-43.

28. Malafa M, Margenthaler j, Webb B, et al. MnSOD expression is increased in metastatic gastric cancer. J Surg Res 2000; 88: 130-4.

29. Ni J, Mei M, Sun L. Oxidative DNA damage and repair in chronic atrophic gastritis and gastric cancer. Hepatogastroenterology 2012; 59: 671-5.

30. Hwang TS, Choi HK, Han HS. Differential expression of manganese superoxide dismutase, copper/zinc superoxide dismutase, and catalase in gastric adenocarcinoma and normal gastric mucosa. Eur J Surg Oncol 2007; 33: 474-9.

31. Monari M, Foschi J, Calabrese C, et al. Implications of antioxidant enzymes in human gastric neoplasms. Int J Mol Med 2009; 24: 693-700

32. Izutani R, Katoh M, Asano S, et al. Enhanced expression of manganese superoxide dismutase mRNA and increased TNFalpha mRNA expression by gastric mucosa in gastric cancer. World J Surg 1996; 20: 228-33.

33. Hempel N, Carrico PM, Melendez JA. Manganese superoxide dismutase (SOD2) and redox-control of signaling events that drive metastasis. Anticancer Agents Med Chem 2011; 11: 191-201.

34. Tsuji T, Ibargi S, Hu GF. Epithelial-mesenchymal transition and cell cooperativity in metastasis. Cancer Res 2009; 69: 7135-9.

35. Nathan CF. Neutrophil activation on biological surfaces. Massive secretion of hydrogen peroxide in response to products of macrophages and lymphocytes. J Clin Invest 1987; 80: 1550-60.

36. Kruidenier L, Kuiper I, van Duijn W, et al. Differential mucosal expression of three superoxide dismutase isoforms in inflammatory bowel disease. J Pathol 2003; 201: 7-16.

37. Hil MJ, Morson BC, Bussey HJ. Aetiology of adenoma-carcinoma sequence in large bowel Lancet 1978; 1: 245-7.

38. Shinya H, Wolff WI. Morphology, anatomic distribution and cancer potential of colonic polyps. Ann Surg 1979; 190: 679-83.

39. Gilles RJ, Robey I, Gatenby RA. Causes and consequence of increased glucose metabolism of cancers. J Nucl Med 2008; 49: 24S-42S.

40. Vander Heiden MG, Cantley LC, Thompson CB. Understanding the Warburg effect: the metabolic requirements of cell proliferation. Science 2009; 324: 1029-33.
41. Newsholme EA, Crabtree B, Ardawi MS. The role of high rates of glycolysis and glutamine utilization in rapidly dividing cells. Biosci Rep 1985; 5: 393-400.

42. Gatenby RA, Gilles RJ. Why do cancers have high aerobic glycolysis? Nat Rev Cancer 2004; 4: 891-9.

Received: 20.06.2015

Accepted: 20.07.2015 